\title{
Simulation and experimental investigation of beam distortions in end-pumped laser rod amplifiers
}

\author{
Philipp Albrodt,${ }^{1 *}$ Xavier Delen, ${ }^{1}$ Mondher Besbes, ${ }^{1}$ Fabien Lesparre, ${ }^{1,2}$ \\ PATRICK GEORGES, ${ }^{1}$ \\ ${ }^{1}$ Laboratoire Charles Fabry, Institut d'Optique Graduate School, CNRS, Université Paris-Saclay, 91127 Palaiseau Cedex, France \\ ${ }^{2}$ Fibercryst SAS, Parc d'activité Wilson Bât A1, 31 Rue Wilson, 69150 Decines Charpieu, France \\ *Corresponding author: philipp.albrodt@institutoptique.fr
}

Received XX Month XXXX; revised XX Month, XXXX; accepted XX Month XXXX; posted XX Month XXXX (Doc. ID XXXXX); published XX Month XXXX

\begin{abstract}
Power scaling of end-pumped laser rod amplifiers is challenging due to beam distortions at high powers. It is difficult to understand these distortions since several physical effects contribute at the same time. We present a detailed investigation of the beam distortions by numerical simulation and experiments in different gain regimes (passive regime with no gain, small signal gain regime, saturated gain regime) in order to decouple effects caused by thermal lensing from aberrations induced by the transversal gain profile. Simulation and experiment are in good agreement and show the influence of the gain regime in details and reveal a significant impact of the transversal gain profile on the output beam quality. (c) 2018 Optical Society of America
\end{abstract}

OCIS codes: (140.3280) Laser amplifiers; (140.3480) Lasers, diode-pumped; (140.6810) Thermal effects; (140.3295) Laser beam characterization;

\section{INTRODUCTION}

Ultrafast lasers have become an indispensable tool for scientific and industrial applications over the last decade [1]. In this field, Master oscillator power amplifier (MOPA) systems are an attractive alternative to high power oscillators and are widely used in optical science [2]. Yb-doped diode pumped solid-state lasers in MOPA configuration are used to achieve high average power ultrafast lasers. There are several different technologies available concerning the geometry of the amplifying medium. Amplifiers based on slabs [3] or thin-disks [4] have reached the kilowatt regime but their architectures are quite complex. Therefore, simple longitudinally pumped laser rod architectures are still of interest for intermediate output power ranges of a few tens of watts. One main advantage is their particularly simple and robust geometry [5]. They can for example be used in laser chains to close the gap between a low power oscillator and a complex highpower amplifier providing a low gain. However, power scaling is usually limited due to thermally induced beam distortions [6]. One main challenge in the recent development of end-pumped laser rod amplifiers is to maintain a good beam quality at high power levels. The degradation of the beam quality results in distorted beam profiles leading to an increased beam quality factor $\mathrm{M}^{2}$. The distorted beam profiles usually vary along the caustic. One typical example is a donutlike intensity distribution [7] which can be observed in the far field. Beam shapes like this can for example be induced by spherical aberration introduced by the thermal lens [8-12]. It is well known that the beam quality can be significantly degraded due to aberrated thermal lensing $[13,14]$, but there are several points, that haven't been studied yet in details. One interesting aspect is the interaction of amplification effects with effects related to aberrated thermal lensing. The gain influences the propagation of the signal through the crystal $[15,16]$ and can therefore change the response of the signal depending on the gain regime. For example, it was shown in previous work, that the seed signal beam quality can be improved in endpumped bulk amplifiers, if the seed signal beam-quality is quite low $\left(\mathrm{M}^{2}\right.$ > 2) $[17,18]$. But the dependence of this effect on the transversal amplification profiles resulting from saturation effects remains unclear. Furthermore, the physical origin of the beam quality degradation of a low power seed signal with diffraction limited beam quality in end pumped laser rod amplifiers remains unclear since both aberrated thermal lensing and gain-profile dependent effects contribute at the same time. With this paper we contribute to a more detailed understanding of the observed beam quality degradations in the saturated gain regime, which is important for ongoing work on laser rod amplifiers with improved output beam quality at high powers. The methods for the investigation of beam distortions proposed in this paper are in general applicable for many kinds of end-pumped bulk amplifiers. We chose to focus on the investigation on Yb:YAG Single Crystal Fibers (SCFs) in the framework of this paper and verified the methods for this technology. SCFs are thin moderately doped laser rods with a diameter of about one millimeter and a length of a few centimeters. This technology has been developed during the last decade [21-23] and has recently shown a promising potential for the 
amplification of ultrashort pulses [24-26]. The good thermal management and the cubic crystal structure of Yb:YAG allow the study of higher order beam distortions for a simplified case.

In the two first sections, we introduce our numerical and experimental tools. Our numerical simulation tools include a rate equation solver to calculate absorption and gain distribution, a Finite Element Analysis (FEA) simulation to evaluate temperature and stress distribution and we use a Beam Propagation Method (BPM) code to study the induced beam distortion. Our experimental setup uses two probe beams at different wavelengths to study the effect of the amplification on beam distortion. Detailed comparisons between our experimental and simulated results are presented in section 4 . We study the physical origin of beam distortion and focus on the effect of gain saturation in the amplifier $[19,20]$.

\section{Theory and numerical methods}

In this section we present the developed numerical methods for the simulation of a single pass of a signal through an end-pumped laser rod amplifier. We are interested in the simulation of steady state operation points and therefore ignore time dependent effects. Several simulation steps are necessary to solve the problem. First, we calculate the absorption of the pump light and the corresponding inversion of population in the laser rod. With this information one can make a good approximation of the heat deposition in the crystal [27]. In this way, one can use Finite Element Analysis (FEA) to calculate the thermomechanic state of the end-pumped rod. These models are described in the following subsection A. After that, the results of these models are used as start parameters for the Beam Propagation Method (BPM), which will be discussed in subsection $B$.

\section{A. Pump absorption and Finite Element Analysis}

We assumed that the transversal pump beam profile is a top-hat-like supergaussian function and not influenced by the pump absorption and the thermal lens. The material properties at $\mathrm{T}=300 \mathrm{~K}$ used in our numerical models are summarized in Tab. 1. Furthermore, the temperature dependence of the absorption and emission cross sections is taken into account as described in [29, 28]. With these values, one can calculate the pump absorption and signal amplification in the SCF by solving the population equations of the four-level system. In general, the pump intensity $I_{p}$ is described by the simple differential equation $d I_{p} / d_{z}=-\alpha_{p} I_{p}$ The absorption coefficient is in our case dependent on the radial and the longitudinal position in the crystal respectively $r$ and $z$. One can express $\alpha_{p}$ with the parameters listed in

Tab. 1 as [31]

Tab. 1 Material properties of $4 \%$ at. Yb:YAG at 300K

\begin{tabular}{lcccc}
\hline & symbol & value & unit & Ref \\
\hline lifetime for excited level & $\tau$ & 0.95 & $\mathrm{~ms}$ & {$[28]$} \\
absorption wavelength & $\lambda_{p}$ & 940 & $\mathrm{~nm}$ & {$[9]$} \\
laser wavelength & $\lambda_{l}$ & 1030 & $\mathrm{~nm}$ & {$[9]$} \\
absorption cross section at $\lambda_{p}$ & $\sigma_{a b s}\left(\lambda_{p}\right)$ & $0.75 \times 10^{-20}$ & $\mathrm{~cm}^{2}$ & {$[29]$} \\
emission cross section at $\lambda_{p}$ & $\sigma_{e m}\left(\lambda_{p}\right)$ & $0.15 \times 10^{-20}$ & $\mathrm{~cm}^{2}$ & {$[30]$} \\
absorption cross section at $\lambda_{l}$ & $\sigma_{a b s}\left(\lambda_{l}\right)$ & $0.16 \times 10^{-20}$ & $\mathrm{~cm}^{2}$ & {$[29]$} \\
emission cross section at $\lambda_{l}$ & $\sigma_{\mathrm{em}}\left(\lambda_{l}\right)$ & $2.1 \times 10^{-20}$ & $\mathrm{~cm}^{2}$ & {$[30]$} \\
density of $\mathrm{Yb}^{3+}$ Ions & $\mathrm{N}_{\mathrm{t}}$ & $5.51 \times 10^{-26}$ & $\mathrm{~m}^{-3}$ & {$[9]$} \\
\hline
\end{tabular}

$$
\alpha_{p}(r, z)=\frac{I_{l}(r, z) N_{t} \tau \times\left[\sigma_{a b s}\left(\lambda_{p}\right) \sigma_{e m}\left(\lambda_{l}\right)-\sigma_{a b s}\left(\lambda_{l}\right) \sigma_{e m}\left(\lambda_{p}\right)\right]+\sigma_{a b s}\left(\lambda_{p}\right) N_{t}}{1+\frac{I_{p}(r, z)}{I_{p, s a t}}+\frac{I_{l}(r, z)}{I_{l, s a t}}}
$$

where, $I_{l}, I_{p}, I_{l, s a t}$ and $I_{p, s a t}$ are respectively the laser intensity, the pump intensity and the corresponding saturation intensies all expressed in photonic units. Saturation intensities are defined by

$$
I_{p, s a t}=\frac{1}{\tau \times\left[\sigma_{a b s}\left(\lambda_{p}\right)+\sigma_{e m}\left(\lambda_{p}\right)\right]}
$$

and

$$
I_{l, s a t}=\frac{1}{\tau \times\left[\sigma_{a b s}\left(\lambda_{l}\right)+\sigma_{e m}\left(\lambda_{l}\right)\right]} .
$$

Saturation intensities can easily be reached in typical end-pumped laser rod amplifiers. This is an important point to keep in mind for the characterization of beam distortions. The absorption of the pump light depends consequently on the intensity distribution of pump and laser light in the crystal. Similar to that, the gain coefficient is influenced by saturation effects in the same way and can be written as

$$
g_{l}(r, z)=\frac{I_{p}(r, z) N_{t} \tau \times\left[\sigma_{a b s}\left(\lambda_{p}\right) \sigma_{e m}\left(\lambda_{l}\right)-\sigma_{a b s}\left(\lambda_{l}\right) \sigma_{e m}\left(\lambda_{p}\right)\right]-\sigma_{a b s}\left(\lambda_{l}\right) N_{t}}{1+\frac{I_{p}(r, z)}{I_{p, s a t}}+\frac{I_{l}(r, z)}{I_{l, s a t}}} .
$$

Given these equations one can calculate the absorption of the pump and signal amplification in the crystal. The heat source distribution in the rod can then be estimated and the thermomechanical steady state can be calculated using FEA.

We used an open source mesh generator [32] and MATLAB®. As a boundary condition for the cooling we defined a fixed heatsink temperature $(300 \mathrm{~K})$ at the side and cooling by convection in room temperature air at the ends of the crystal. The thermomechanical properties of Yb:YAG were taken from [9]. Then we used standard FEA calculation methods, following [33], to calculate the three dimensional temperature, strain and stress distribution in the rod. The corresponding thermally and mechanically induced OPD can then be calculated.

In order to illustrate this part, we present in the following some fundamental simulation results for one specific operating point that corresponds to a standard operating point of the experimental setup, which will be described later in section four. We considered a Yb:YAG SCF (length $l=40 \mathrm{~mm}$, diameter $\varnothing=1 \mathrm{~mm}$, doping concentration $d_{c}=4$ $\%$ at. ) and a top-hat-like pump beam with a diameter of $\emptyset_{\mathrm{p}}=350 \mu \mathrm{m}$ at the waist with an optical power of $P p=126 \mathrm{~W}$, which corresponds to the maximum pump power level used in the experimental setup. The signal diameter was chosen at $\emptyset_{s}=350 \mu \mathrm{m}$, which is a standard value for this type of end-pumped SCF amplifiers.

It is interesting to study the dependence of the OPD and the considered seed power level. For the case of no incident signal, the heat source is constant within the pump radius and the temperature distribution in the crystal can be described by analytical equations. The definition of the heat source gets more complex under the presence of a signal and it becomes necessary to use the numerical models discussed above. The calculated OPD as a function of the crystal radius is shown in Fig. 1 for a few different seed power levels. We assumed a linear dependence (with $d n / d T=7.3 \times 10^{-6} K^{-1}$ ) $[34,35]$ of the refractive index on the local temperature. We neglect any stress dependent effects and the deformation of the crystal facets since previous work has shown, that these effects are much less important for our case $[31,36]$, in particular 
for the SCF having a high aspect ratio (crystal length / crystal diameter). Additionally, we neglect polarization depend effects as bifocusing or depolarization have not been observed in recent work on SCF amplifiers [24-26]. One can see in Fig. 1 that the overall phase differences increases with the seed power level linked to an increasing temperature gradient caused by a concentration of the corresponding heat source close to the surface under the presence of significant seed signal. One interesting thing to look at concerning these calculated OPD is the resulting non-parabolic phase, which is accumulated by a signal propagating through the end-pumped crystal. The central region of the profiles shown in Fig. 1 can be approximated accurately by a parabola.

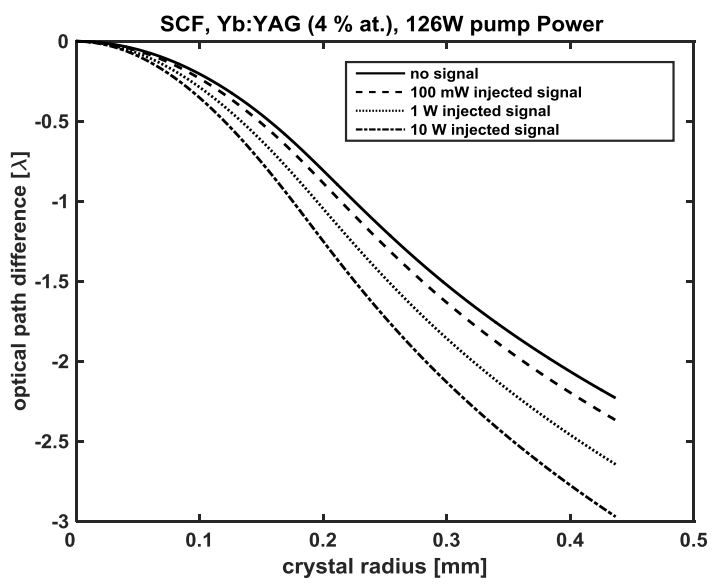

Fig. 1 Calculated OPD for an Yb:YAG SCF at $\left(P p=126 \mathrm{~W} ; P_{i n}=\{0 ; 0.1 ; 1\right.$; $\left.10\} \mathrm{W}, \varnothing_{s}=\varnothing_{p}=350 \mu \mathrm{m}\right)$

The difference of the OPD to such a parabolic fit is shown in Fig. 2 and represents the non-parabolic phase finally leading to a decreasing beam quality. The non-parabolic phase is restricted to radii exceeding the pump radius for low seed power levels. But for higher seed power levels, this is no longer the case, since the non-parabolic phase is remarkably different from zero even inside the pumped area at higher seed power levels.

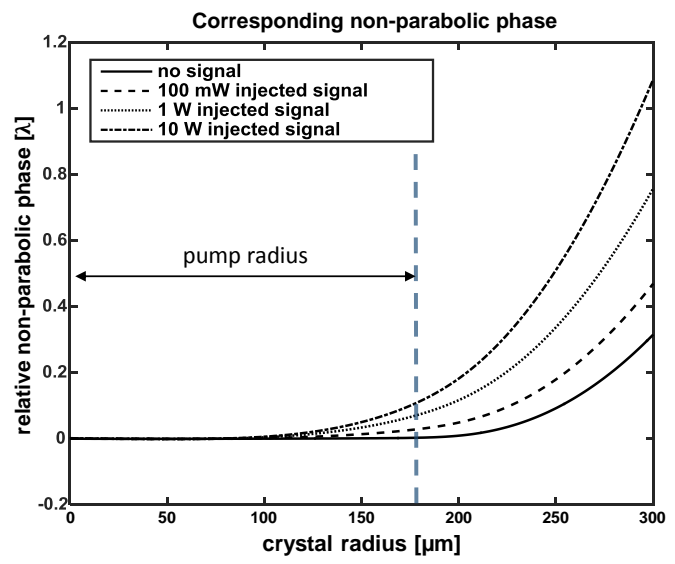

Fig. 2 Corresponding non-parabolic phase of the OPDs shown in Fig. 1.

We projected these calculated non-parabolic phase profiles on the Zernike basis [37] and evaluated the coefficient $Z_{4}^{0}$ for primary spherical aberration at many different seed-power levels. Fig 3 shows the calculated coefficients for a seed power range from $0.1 \mathrm{~mW}$ to $100 \mathrm{~W}$. The results are normalized on the calculated coefficient for the smallest signal. One can see that the variations in the milliwatt regime are small and can be ignored. In contrast to that, the amount of induced spherical aberration rapidly increases for seed powers higher than $100 \mathrm{~mW}$ and can be 2.5 times higher in comparison to the small signal gain regime. Our Simulations indicate a decreasing amount of spherical aberration for seed signals higher than $50 \mathrm{~W}$, where the absorption of the pump light is close to a top-hat-like profile over the pumped aperture. It can be understood considering the signal transition starts to saturate even at the outer part of the pump beam. However, this effect becomes relevant for signal input powers in the range of the applied pump power and therefore not relevant for the design of amplifiers.

These preliminary simulations show that a strong influence of the gain saturation regime on beam distortion can be expected. Beam distortions should increase as we go from small signal gain regime to saturated gain regime.

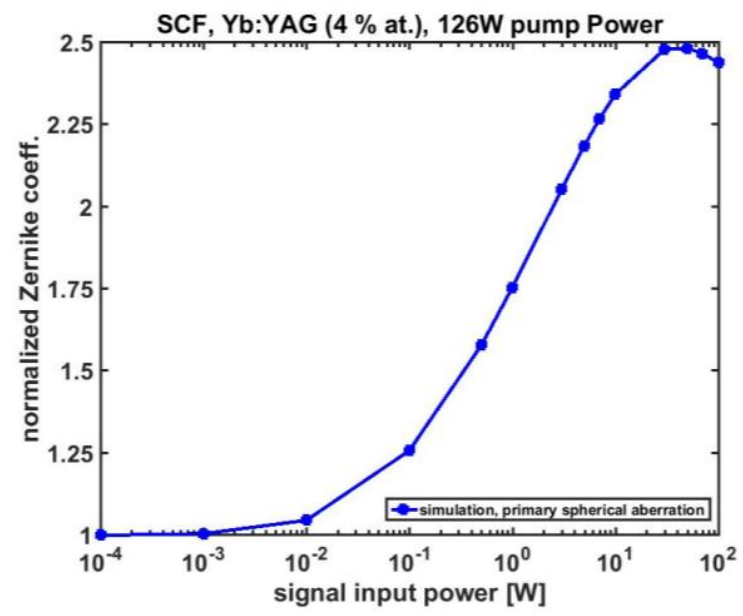

Fig. 3 calculated primary spherical aberration for various seed power levels $\left(P p=126 \mathrm{~W}, \varnothing_{s}=\varnothing_{p}=350 \mu \mathrm{m}\right)$

The theory and the numerical methods presented up to this point allow to calculate the thermomechanical state of the crystal. These results are hard to compare to experiments. In the following section, we present the simulation of the propagation of the seed laser through the crystal. This simulation step allows to calculate distorted beam profiles and the corresponding beam quality degradation, which can later be compared to experimental results of beam quality measurements as described in section 4 .

\section{B. Beam Propagation Method}

The numerical calculation of beam propagation using the principles of Fourier Optics is often referred to as the BPM. The principles of Fourier Optics have to be applied in order to study the effect of a complex thermal gradient on the propagation of laser beams. The general principles of these propagation methods are thoroughly documented in the literature [38, 39]. In our case, we are working in a regime where the Fresnel approximation [40] is an adequate approximation for the propagation. However, the signal propagation through a non-uniform optical medium remains non-trivial. The crystals of end-pumped laser rod amplifiers are usually a few centimeters long and the influence of the amplification and the induced phase-distortions on the signal propagation have to be considered. We consequently used a stepwise calculation method. Here, the nonuniform medium is divided into a large number of segments (see Fig. 4), each having a centered effective plane. In this way the output beam quality field distribution $\boldsymbol{u}_{\boldsymbol{o u t}}(\boldsymbol{x}, \boldsymbol{y})$ is computed by propagating the 
input field $\boldsymbol{u}_{\boldsymbol{i n}}(\boldsymbol{x}, \boldsymbol{y})$ stepwise while the signal is modulated in phase and amplitude at each effective plane. Similar propagation models have been used by others earlier in order to simulate the improvement of the beam quality for seed laser beams with a quite low beam quality [17]. The modulation at the effective plane is

$$
u_{j}^{\prime}(x, y)=u_{j}(x, y) \times \exp \left[-i \varphi_{j}(x, y)\right] \times \exp \left[\frac{g_{j}(x, y)}{2} \Delta z\right],
$$

where the effective phase $\varphi_{i}(x, y)$ and gain coefficient $g_{i}(x, y)$ are calculated using the results of the previously mentioned simulation models and $\Delta \mathrm{z}$ is the step size. After propagation through the crystal, the signal is propagated through an imaging system in order compute the output field in several different observation planes along the caustic. Our calculated intensity distributions can then be easily compared to measurement results. Finally, the second momentum beam width in several planes and the $M^{2}$-Parameter are calculated as it is usually done experimentally. The results of the BPM are presented and compared to experimental results in section 4 .

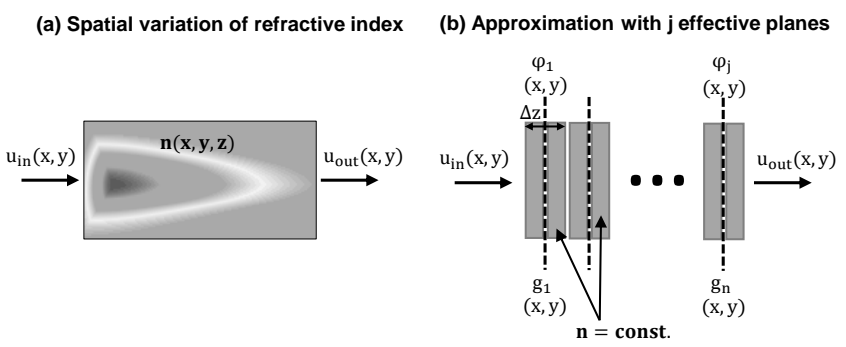

Fig. 4. Sketch of the applied approximation to simulate the propagation of a signal through a medium with spatially varying optical properties

\section{EXPERIMENTAL SETUP}

Fig. 5 shows the experimental setup of the investigated amplifier. A single pass in contra-propagation pump configuration through the SCF is investigated. Usually end-pumped laser rod amplifiers are used in a double pass configuration in order to reach higher extraction efficiencies. However, the investigation of a double pass can be quite complex and is not discussed in this paper. The injected seed signal can be switched between two wavelengths inside or outside the gain bandwidth. This is essential for the investigation of the beam distortions in different regimes of the amplifier. The amplified signal was chosen at $\lambda_{l}=1030 \mathrm{~nm}$ where the spectrum of the emission crosssection for Yb:YAG reaches its highest value which leads to a strong amplification of the signal. The passive probe beam wavelength in the experimental setup is $\lambda_{p}=1064 \mathrm{~nm}$ at which the emission cross section is more than one order of magnitude lower [41] compared to the amplified signal. This leads to an absolute value of the gain which is much lower in comparison to the amplified signal and we can therefore neglect the amplification at this wavelength and use this beam as a passive probe. It allows to study the behavior of the laser amplifier with no relevant injection of the laser wavelength. The two beams are overlapped with the pump beam in the amplifier using dichroic mirrors (DM). The output signal quality is characterized using a wavefront analyzer and a measurement of the $\mathrm{M}^{2}$ parameter. The size and position of the seed signal waist can be adjusted by an optical zoom. In this way one can change the beam size ratio $\beta=\emptyset_{s} / \emptyset_{p}$ easily, with the signal/pump-diameter $\emptyset_{s, p}$. Previous studies showed that $\beta$ is an important parameter regarding the output beam quality [14]. The seed power level of the seed beams is in the range of a few $\mathrm{mW}$, which corresponds well to the small signal regime as mentioned earlier. The behavior of the amplifier is close to the small signal gain regime for those operating points. In order to study the saturated gain regime, the fiber coupled laser source in Fig. 5 is replaced by a highpower laser system delivering up to $10 \mathrm{~W}$ at $1030 \mathrm{~nm}$.

To summarize, we can measure the beam quality degradation with this setup for non-amplified probe signals, and for amplified signals both in the small signal gain regime and the saturated gain regime. We present experimental results compared to the corresponding simulations in the next section.

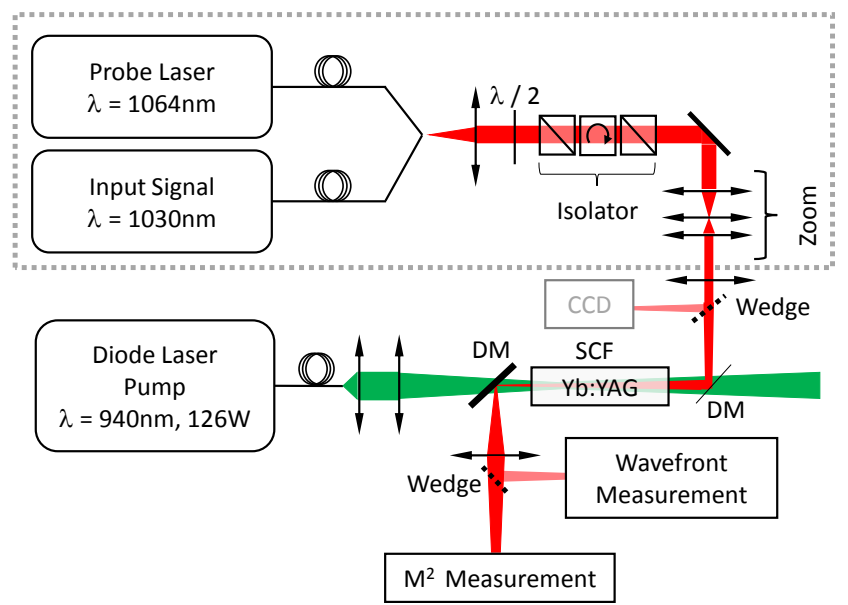

Fig. 5. Simplified sketch of the experimental setup used for the characterization of beam distortions

\section{CHARACTERISATION}

In this section we present our characterization of beam distortions in different amplification regimes. The point of operation is specified by the applied pump power $P_{p}$, the seed signal power $P_{\text {in }}\left(P_{i n}=0\right.$ if the probe laser source was used), the diameter of the pump spot $\varnothing_{p}$ and the diameter of the passive probe or amplified signal laser beam $\emptyset_{s}$

We discuss the beam quality degradation for non-amplified probe signal in subsection A. We present a comparison of simulation and measurements of the phase profile; the characteristic beam distortions and the calculated and measured $M^{2}$ parameters in order to verify the developed simulation tools. After that, we study the small signal gain regime and make use of the simulation tools to get a better understanding of the measured experimental results. Finally, we describe the beam quality degradation in the saturated gain regime in subsection $\mathrm{C}$ by using the simulation tools to estimate the influence of the transversal gain profile and present measurements of the beam profiles and the $M^{2}$ parameter at different seed power levels.

\section{A. Beam quality degradation for non-amplified probe signals}

We study the regime without amplification in order to decouple the effect of the thermal lens from the effect of amplification on the beam quality degradation. This regime is a good starting point to verify the developed simulation tools. We present in the following simulation and measurement of the phase profile of the thermal lens at a high pump power level, the corresponding beam deformations to this operating point and measurement and simulation of the $\mathrm{M}^{2}$ parameter at different pump power levels.

\section{Phase measurements}



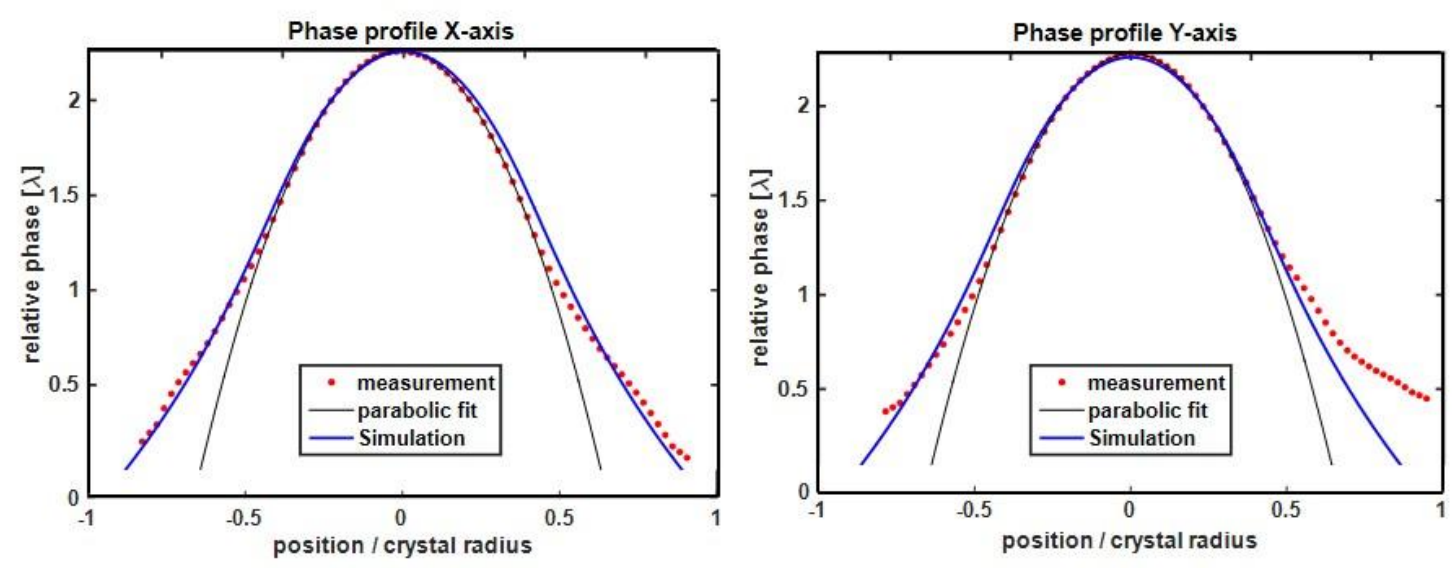

Fig. 6 Measured and simulated phase profiles of the probe signal: (left) profile parallel to $x$-axis and (right) profile parallel to y-axis. The beam radius $(4 \sigma)$ in the measurement plane was $0.5 \mathrm{~mm} .\left(P_{p}=126 \mathrm{~W}, \varnothing_{s}=400 \mu \mathrm{m}, \varnothing_{p}=350 \mu \mathrm{m}, P_{\text {in }}=0 \mathrm{~W}\right)$

We used a wavefront sensor based on the principle of quadriwave lateral shearing interferometry [42]. The accuracy of the sensor was better than $10 \mathrm{~nm}$, corresponding approximately to $\lambda / 100$. Measured and simulated phase profiles of the passive probe are illustrated in Fig. 6 . The signal diameter exceeded the pump spot diameter significantly here. In this way, the intensity on the aberrated outer area is high enough to be detected with the wavefront sensor. We used the passive probe signal for this verification in order to avoid the influence of the gain profile on the detected signal. In this case, the measured phase is directly linked to the thermally induced optical path difference (OPD) in the crystal.

Both measured and simulated phase profile are mostly parabolic in the center and show a logarithmic-like tail at the outer areas of the beam. The parabolic part can be seen as a defocus of the input beam and does not influence the beam quality. The tails differ considerably from the parabolic fit, which means that they are inducing higher order geometrical aberrations, for example primary spherical aberration. The results of simulation and measurement shown above are in satisfying agreement. The slight difference of simulation and the measurement profile on the right side are limited to the outer regions of the beam and were probably caused by slight asymmetries in the experimental setup.

\section{Beam deformations}

We compared the corresponding beam deformations for the measured and simulated phase shown in Fig. 6. To make the effect clearly visible, we investigated an extreme case, with a sufficiently large probe beam size $(\beta \approx 1.14)$. Here, the beam is usually heavily distorted and typical beam deformations can be observed. Again we used the passive probe. We found similar beam distortions along the caustic after the waist for simulation and experiment (see Fig. 7).

We found heavily distorted beam profiles showing a sequence of characteristic profiles in simulation and experiment. While the beam profile at the focus remains quite clean, a halo-like intensity distribution develops shortly after the focus. This effect evolves until a donut-like intensity distribution is clearly visible. And finally a structure of concentric rings appears. This sequence of beam profiles is characteristic for the type induced phase distortions presented earlier and is mainly caused by spherical aberration. Considering the presented measurement of the phase profile and the corresponding beam deformations one can say that the beam quality degradation can be modeled accurately by our simulation tools. For other operational points, we also compared the simulated and measured $\mathrm{M}^{2}$ parameter. (a)
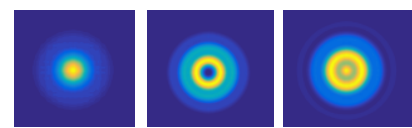

(b)

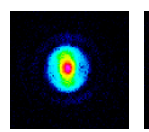

Fig. 7 Simulated (a) and measured (b) intensity distributions of observation planes showing characteristic beam distortions for the phase profiles shown earlier in Fig. $6\left(P_{p}=126 \mathrm{~W}, \varnothing_{s}=400 \mu \mathrm{m}, \varnothing_{p}=\right.$ $350 \mu \mathrm{m}, P_{i n}=0 \mathrm{~W}$ )

\section{Beam quality parameter}

In the previous subsection, we investigated an extreme case $(\beta>1)$ to obtain beam profiles which are easy to identify. To verify the models at different operating points, we considered a more realistic case $(\beta=1)$ and compared the measured and simulated $\mathrm{M}^{2}$ parameter at different pump power levels. The results summarized in Fig. 8 show a good agreement between simulation and experiment. Note that these results were obtained for the passive probe, where the amplification in the BPM was ignored. The decreasing beam quality at increasing pump power levels shown in Fig. 8 shows the influence of the aberrated thermal lens at different pump power levels. One can clearly say that if there is no amplification, the highest tolerable pump power level where one can expect a satisfying beam quality is quite limited. However, this is only one part of the full picture, since the signal amplification can have an important influence on this trend. We therefore analyze the beam quality degradation in end-pumped laser rod amplifiers in the presence of optical gain in the following sections. 


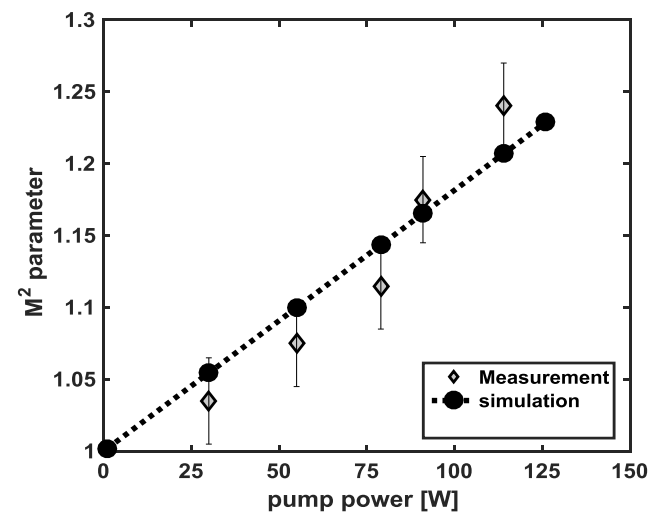

Fig. 8 Measured and simulated BQ parameter of the passive probe at various pump powers $\left(\varnothing_{\mathrm{s}}=\emptyset_{\mathrm{p}}=350 \mu \mathrm{m}, \mathrm{P}_{\mathrm{in}}=0 \mathrm{~W}\right)$.

\section{A. Beam quality degradation in the small signal gain regime}

The thermal load and the resulting OPD in the crystal is almost identical for the investigation of a highly amplified signal under small signal gain regime and a passive probe signal. As a result, a small amplified signal and the passive probe are modulated in their phase almost identically. However, the amplitude modulation is very different (high gain for the $1030 \mathrm{~nm}$ signal in the small signal gain regime). We investigated the response for those two configurations in simulation and experiment in order to determine the influence of the signal amplification on the resulting beam quality.

The measured beam quality of a small amplified signal is given in Fig 9. A reference measurement was done for the passive probe at equal test conditions. The injected seed signal power was $1 \mathrm{~mW}$ for both cases leading to a gain of about $23 \mathrm{~dB}$ at the investigated pump power level of $114 \mathrm{~W}$. The seed lasers were fiber coupled single mode DFBlaser diodes and the beam quality at the entrance facet of the SCF was nearly diffraction limited $\left(M^{2}<1.1\right)$. It was verified that the waist of the seeded beam was well overlapped with the pump beam waist and that the beam size ratio was $\beta \approx 1$. One can see that the measured beam quality of the amplified signal remained nearly diffraction limited $\left(M^{2}<\right.$ 1.1). In contrast to that, the reference measurement done with the passive probe at equal test conditions showed a considerably decreased beam quality $\left(M^{2} \approx 1.24\right)$. While the beam shapes of the amplified signal remained close to an ideal Gaussian beam along the caustic, characteristic beam distortions (see halo-like and donut-like beam shape in Fig. 9) were observed for the passive probe. Given these points, it is clear that the response of the strongly amplified signal is quite different to the response of the passive probe.

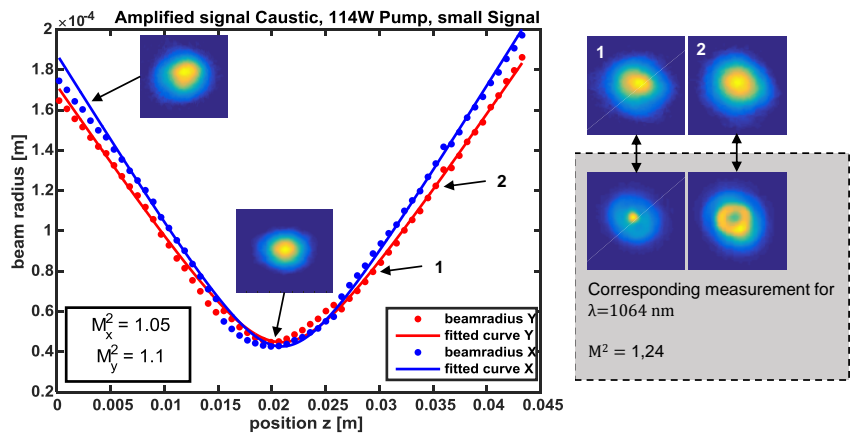

Fig. 9 Measured caustic and intensity distributions for a small amplified signal. The corresponding measurement for the passive probe was done at equal test conditions: $P_{\text {in }}=1 \mathrm{~mW} ; \emptyset_{s}=\emptyset_{p}=350 \mu \mathrm{m} ; P_{p}=114 \mathrm{~W}$.

This effect can be reproduced by our simulations. The results summarized in Fig. 10 were calculated for an extreme case with a critical beam size ratio $(\beta \approx 1.14)$ at $126 \mathrm{~W}$ Pimp power in order to make the effect clearly visible. We found a considerably different response of the passive probe and amplified signal beam. While the amplified signal remains close to the diffraction limit $\left(M^{2} \approx 1.1\right)$, the calculated beam quality of the passive probe is considerably decreased $\left(M^{2}<1.5\right)$. The characteristic deformations of the intensity distribution were observed for the passive probe, while the beam shapes of the amplified signal remained nearly Gaussian. The position where the characteristic donut-like beam shape was calculated is marked in Fig. 10 . The results for this simulation study were experimentally verified. While the measured beam quality of the passive probe was poor $\left(M^{2} \approx\right.$ 1.4) and the observed beam deformations quite strong (see Fig. 7(b)), a nearly diffraction limited $\left(M^{2} \approx 1.1\right)$ beam quality was measured for the amplified signal.
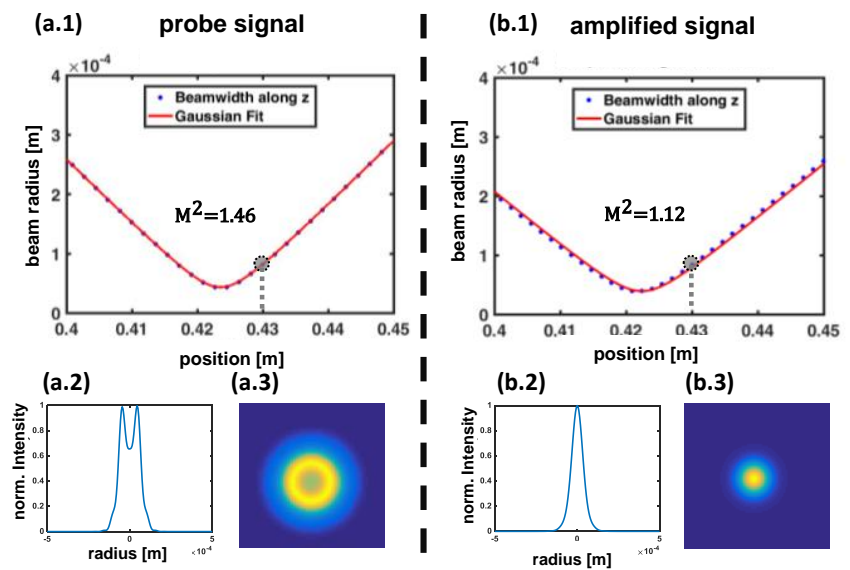

Fig. 10 Numerically calculated caustic (1) and intensity profile (2) and distribution (3) at the marked position $(\mathrm{z}=0.43 \mathrm{~m})$ for (a) the passive probe and (b) the amplified signal, with: $P_{i n}=300 \mathrm{~mW} ; \emptyset_{s}=400 \mu \mathrm{m} ; \emptyset_{p}=350 \mu \mathrm{m} ; P_{p}=126 \mathrm{~W}$.

Altogether, these investigations show that there is a considerably different response for an amplified and passive seed to a very similar phase modulation. One can see the strong signal amplification in the small-signal gain regime as a spatial filter acting like a soft aperture. The influence of the outer parts, which leads to a decreasing beam 
quality for a passive probe, is suppressed by the amplitude modulation of the small signal gain. It was shown in other research work, that the beam quality of the seed laser beam can be enhanced due to this effect [17]. However, our results add new important details, since we showed that the decreasing beam quality in end-pumped laser rod amplifiers is not only related to the pump density but strongly coupled to the amplification regime.

\section{B. Beam quality in the saturated gain regime}

To go one step further, one has to investigate the regime of higher seed power levels, where the transversal profile of the signal amplification can be significantly different from the small signal gain profile. Several characteristics of the laser amplifier change as a function of the seed power. Firstly the absorbed pump power increases, since the consumption of the inversion of population leads to an increased absorption of the pump beam. This leads to an increasing thermal load $P_{t}$. Secondly the spatial distribution of the thermal load $d P_{t}(r, z)$ is varying. These two effects lead to the variation of the induced non-parabolic phase discussed earlier. Finally the gain level and the spatial distribution of the gain coefficient $g(r, z)$ depend on the seed-power. All these effects together can cause a significant difference of the output beam quality in comparison to the small signal gain regime. These effects can be decoupled and studied independently using numerical simulations.

\section{Simulations}

We calculated the thermal load and the gain distribution for different seed powers at a high pump power level (126 W). It makes sense to present the results on a normalized scale, where conditions of the small signal gain regime are a practical reference point (we chose the characteristics of the amplifier for a $0.1 \mathrm{~mW}$ seed signal as the small signal gain regime). Similar to the experimental setup discussed earlier, the simulation considers contra-propagating pump and signal beam in a single pass configuration. As mentioned above, the parameters are varying in with the radius $r$ and the position $\mathrm{z}$ along the optical axis. We present the calculated results at the pump entrance facet. We chose this specific plane, since it is the plane with the highest densities of pump and signal power and consequently the most critical plane. The density variation of the thermal load at this position is given in Fig. 11.

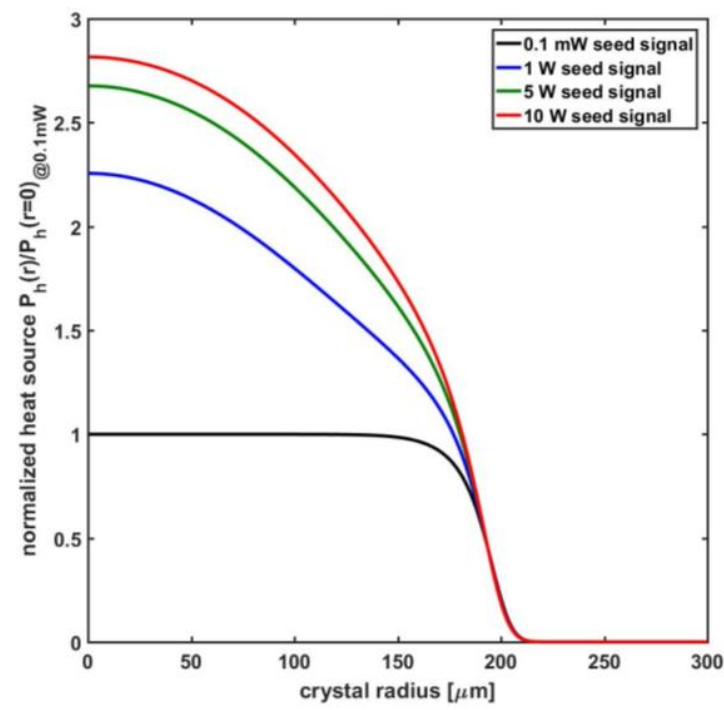

Fig. 11 Calculated radial profiles of the thermal load for different seed power levels $\left(P_{i n}=0.0001-10 \mathrm{~W}, \emptyset_{s}=\emptyset_{p}=350 \mu \mathrm{m}\right)$
The solid black line shows the calculated radial distribution of the effective heat source for the small signal gain regime (the results are normalized on the heat source density at $r=0$ ). For the small signal gain regime, the spatial distribution of the heat source follows the form of the pump intensity, which we defined as a top-hat- like superGaussian distribution. For increasing seed power levels, the integral over the plotted lines increases, which indicates that overall heat power level is increasing. Furthermore, the distribution of the heat source is no longer a top-hat. The red line corresponds to a seed power of $10 \mathrm{~W}$. Here, the difference to the top-hat form is very clear and the maximal density of the heat source is significantly higher than in the small signal gain regime. A similar trend can be observed for $1 \mathrm{~W}$ seed power (blue line) and $5 \mathrm{~W}$ seed power (green line). The considerable change in the shown radial distribution explains, why the calculated OPD of the SCF varies as shown earlier in Fig 1 and 2. While a constant heat source leads to a parabolic phase [9], the induced phase by heat sources similar to the profiles shown in Fig. 11 is not parabolic and can only be calculated with numerical simulations.

The variation of the gain coefficient at the pump entrance facet is equally important. The spatial variation of the gain coefficient was calculated and is shown in Fig 12.

The results are normalized on the corresponding small signal gain coefficient. The radial profile of the gain coefficient for small signals (black line) is again a top-hat-like profile, meaning that the signal is amplified homogeneously in the pumped area. For higher seed power levels, the gain coefficient decrease rapidly and drops to about $25 \%$ of the small signal gain for a $10 \mathrm{~W}$ seed (red line) at $r=0$. Additionaly, the radial profile of the gain distribution is no longer a top-hat and shows a pan-like shape in the central region of the crystal. Therefore, the outer part of the signal beam is more amplified than the central part.

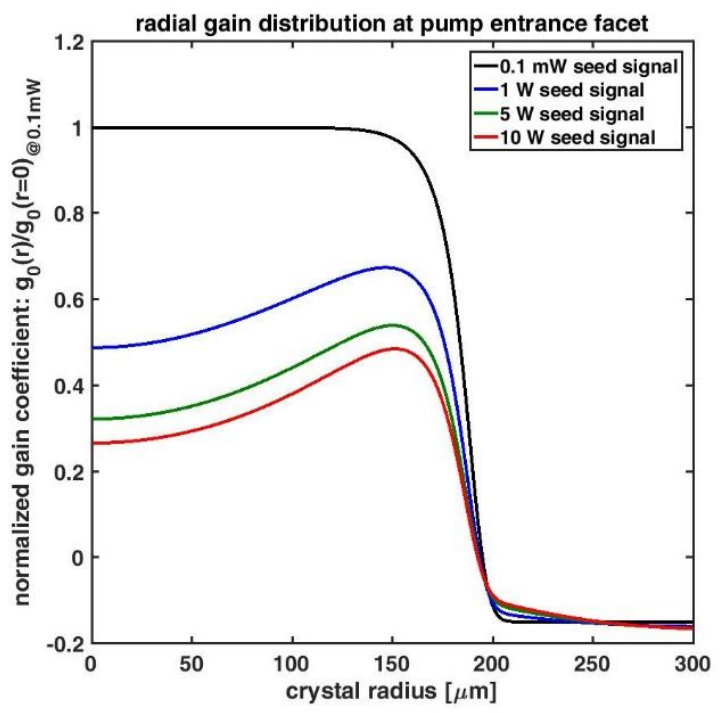

Fig. 12 Calculated radial profiles of the gain coefficient for different seed power levels $\left(P_{i n}=0.0001-10 \mathrm{~W}, \emptyset_{s}=\varnothing_{p}=350 \mu \mathrm{m}\right)$

It is easy to understand that both effects discussed above can lead to a decreasing beam quality in the regime of high extraction powers. However, it is not clear which effect is the most important. We calculated the corresponding beam quality factor for an amplified signal at different seed power levels. We consider therefore four different cases:

(a) variation of $d P_{t}(r, z) ; P_{t}$ and $g(r, z)$ at the same time;

(b) variation of $g(r, z)$ only;

(c) variation of $d P_{t}(r, z)$ only; 
(d) and variation of $P_{t}$ only

For the cases (b) - (d), the remaining parameters were calculated for the case of a small signal for all investigated seed powers. These are of course theoretical cases, but their investigation helps to understand the interaction of the different phenomena in the high-power regime, and their relative influence.

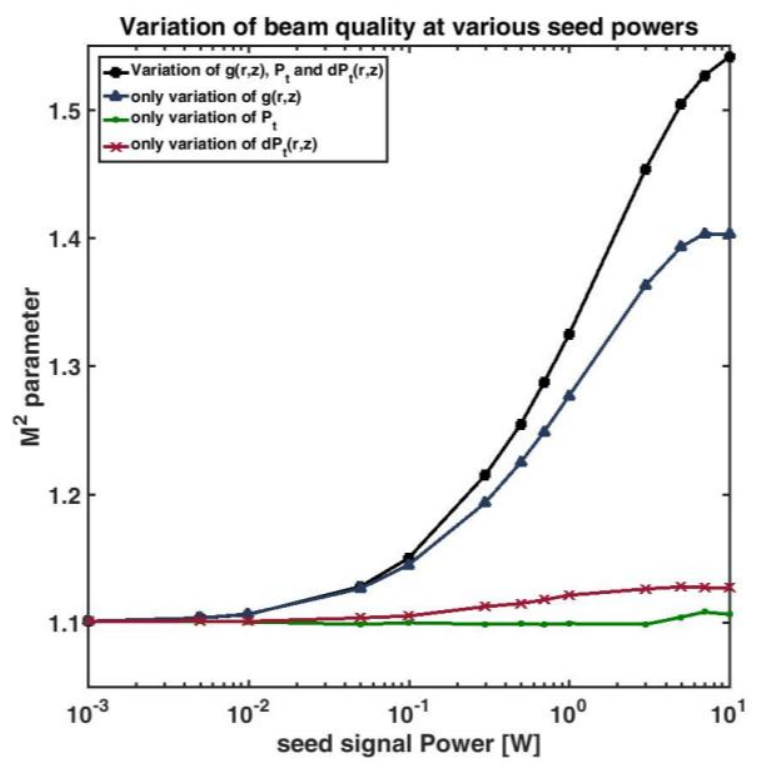

Fig. 13 Calculated radial profiles of the gain coefficient for different seed power levels $\left(P_{i n}=0.0001-10 \mathrm{~W}, \emptyset_{s}=\varnothing_{p}=350 \mu \mathrm{m}\right)$

Fig. 13 shows the calculated output $\mathrm{M}^{2}$ parameter for the four cases described above at various seed-power levels varying from $1 \mathrm{~mW}$ to $10 \mathrm{~W}$. The pump power was $126 \mathrm{~W}$ for all displayed data points. The black line corresponds to case (a) which is the most complicated case but also the most accurate approximation to real amplifiers. One can see that the dependence of the beam quality is highly dependent on the seed power level. While nearly diffraction limited beam quality was calculated for seed powers in the range of a few $\mathrm{mW}$, it is decreasing rapidly for seed powers in the range of a few $\mathrm{W}$. For $10 \mathrm{~W}$ seed power a poor beam quality with $M^{2}>1.5$ was calculated.

The other lines displayed in Fig. 13 illustrate the influence of the decreasing beam quality for the corresponding effects. The calculated effect of the varying dissipated heat power, which is test case (d), is not relevant for the decreasing beam quality. Similar to that, the effect of the varying spatial distribution of the dissipated heat, which is test case (c), is quite weak. One should keep in mind that the gain level and the spatial gain distribution was considered to be the small signal gain distribution for those cases. As discussed earlier, the small signal gain has a considerable influence on the output beam quality and can act as a spatial filter in the crystal. In contrast to that, the influence of the spatial gain variation, which is test case (b), is remarkably strong. At 10 W seed power, a degraded beam quality with $M^{2} \approx 1.4$ was calculated, which explains a large part of the overall decreased beam quality for case (a).

These simulation results underline the strong influence of the gain regime and the spatial gain variation on the output beam quality of end-pumped laser rod amplifiers.

\section{Experimental investigation}

We measured the beam quality at different seed power levels experimentally. Here we added a preamplifier to be able to seed the amplifier at higher powers. We investigated the beam quality therefore at only four different seed power levels: $0.3 \mathrm{~W} ; 1 \mathrm{~W} ; 5 \mathrm{~W}$ and $10 \mathrm{~W}$. Even if the trend of the evolution of the $M^{2}$ parameter shown above in Fig. 14 appears quite clear we decided to use a slightly larger signal size in order to be able to observe a beam quality degradation, which is clearly measurable

The measured intensity distributions are shown in Fig. 14. The images in one row were taken at the same seed-power level (see column on the right) and the images in one column correspond to a

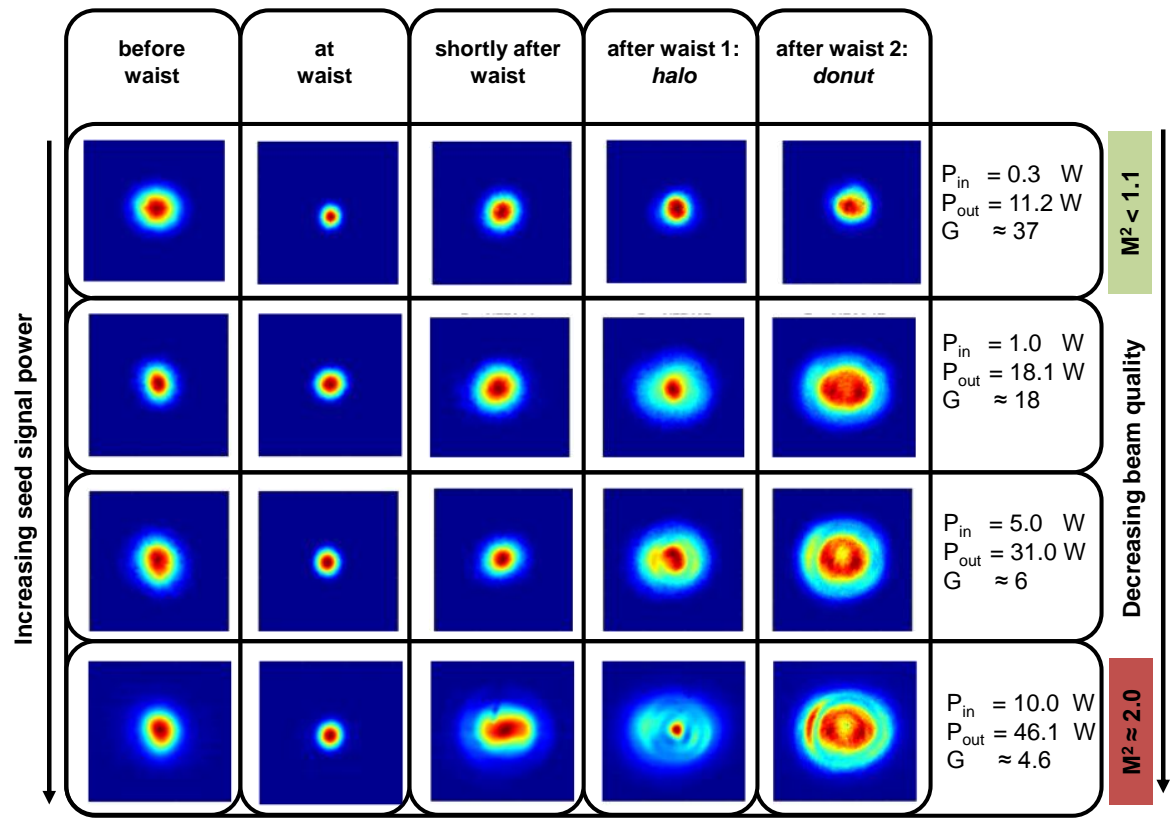

Fig. 14 Measured intensity distributions for different seed power levels at high pump power. $\left(P_{p}=126 \mathrm{~W}, \emptyset_{s}=400 \mu \mathrm{m}, \emptyset_{p}=350 \mu \mathrm{m}\right)$. Seed Laser beam was generated in a preamplifier of a mode-locked oscillator $\left(f_{\text {rep }}=20 \mathrm{MHz}, \tau_{p}=1.1 \mathrm{ps}\right)$. The input signal beam quality of the beam seeded into the experimental setup discussed in this article was nearly diffraction limited $\left(M^{2}<1.1\right)$ 
specific point along the caustic, e.g. the beam waist (see row on the top). Additionally, the seed power level $P_{i n}$, the output power $P_{\text {out }}$, and the overall gain $G=P_{\text {out }} / P_{\text {in }}$ are given for each measurement. One can see that the corresponding beam quality for the lowest seed power level $(0.3 \mathrm{~W}$, see first row) is nearly diffraction limited. The measured beam profiles along the caustic are clean and do not show any characteristic deformations. The $M^{2}$ parameter was close to unity $\left(M^{2}<\right.$ 1.1). The overall signal amplification at this seed-power level is still quite strong $(G \approx 37)$ and the output power level of about $11 \mathrm{~W}$ is low compared to the pump power level (126W). This measurement can be seen as an example of the regime of strong signal amplification and is a good reference point for the other measurements. For the slightly higher seed power of $1 \mathrm{~W}$ one can already observe the decreasing beam quality. The beam profiles after the waist slightly changed to a circular halo (see 4. column in Fig. 14) and the trend to a donut-like profile can be observed in the far-field (see 5. column). This trend becomes clearly visible for a seed-power level of $5 \mathrm{~W}$ (see 3. row). For the highest seed-power level, the beam profiles after the waist are heavily deformed and the overall beam quality was clearly degraded $\left(M^{2} \approx 2\right)$. The overall signal amplification was $G \approx 4.6$ and the output power after a single pass through the amplifier was $46 \mathrm{~W}$. In this regime the extracted power becomes relevant in comparison to the pump power level and the comparably low signal amplification shows that the gain was saturated. For all measurements, the measured beam profile at the waist and shortly before the waist were comparably clean.

Note that we designed the amplifier and this experiment in order to observe a significant distortion of the beam in the saturated gain regime on purpose. At this and higher power levels it is nevertheless possible to achieve nearly diffraction limited beam quality by an adequate design of the amplifier [25,26]. However, further increase of the power is challenging due the typical beam distortions described in this paper.

\section{Conclusion}

Thermal lensing in laser rods is a well-known problem, nevertheless it is difficult to understand the beam quality degradation in end pumped laser rod amplifiers in details, since both the thermal lens and the amplification profile of the amplifier contribute among other effects to the output beam quality at the same time.

In this article, we presented the characterization of beam distortions in end-pumped laser rod amplifiers for different gain regimes (passive regime with no gain, the small signal gain regime and the saturated gain regime) to decouple these effects in order to evaluate the respective contribution of the aberrated thermal lens and the signal amplification.

Firstly, the investigation of passive probe signals showed that characteristic distorted beam profiles (e.g. a donut-like intensity distribution) can be induced by the phase distortions, which are accumulated by the signal propagating through the end-pumped crystal. This simplified case was used to verify the developed numerical methods which are in good agreement with the experiments.

Secondly, the investigation of the small signal gain regime showed that the response of an amplified and a non-amplified signal to the same thermally induced phasemask are significantly different. This effect can be seen as a spatial filtering effect by the transversal gain distribution conserving the input beam quality. Our simulations confirmed these observations.

Finally, the investigation of the high power regime revealed that the changing spatial distribution of the gain is a critical effect regarding the output beam quality of end-pumped bulk amplifiers at high powers.
This effect was investigated theoretically in detail and the experimental observations show the same trend. We observe that one has to pay special attention to the process of the signal amplification while characterizing the beam quality of laser amplifiers.

Given these points, one can say that the physical origin of the observed beam distortions in high power end-pumped laser rod amplifiers can be understood in detail by the investigation presented in this work. The developed numerical simulations can be used in the future for further investigations and the design and test of compensation elements.

\section{References}

1. W. Sibbett, A. A. Lagatsky, and C. T. A. Brown, "The development and application of femtosecond laser systems," Opt. Express 20, 6989 (2012).

2. D. J. Richardson, J. Nilsson, and W. A. Clarkson, "High power fiber lasers: current status and future perspectives [Invited]," J. Opt. Soc. Am. B 27, B63-B92 (2010).

3. P. Russbueldt, T. Mans, J. Weitenberg, H. D. Hoffmann, and R. Poprawe, "Compact diode-pumped $1.1 \mathrm{~kW}$ Yb:YAG Innoslab femtosecond amplifier," Opt. Lett. 35, 4169 (2010).

4. J.-P. Negel, A. Voss, M. A. Ahmed, D. Bauer, D. Sutter, A. Killi, and T. Graf, "1.1 kW average output power from a thin-disk multipass amplifier for ultrashort laser pulses," Opt. Lett. 38, 5442 (2013).

5. D. Sangla, I. Martial, N. Aubry, J. Didierjean, D. Perrodin, F. Balembois, K. Lebbou, A. Brenier, P. Georges O. Tillement, J.-M. Fourmigué, "High power laser operation with crystal fibers", Appl. Phys. B 97, 263-273 (2009).

6. W. Koechner, "Transient thermal profile in optically pumped laser rods" Journal of Applied Physics 44, 3162-3170 (1973).

7. I. Kuznetsov, I. Mukhin, O. Palashov, and K.-I. Ueda, "Thin-tapered-rod Yb:YAG laser amplifier," Opt. Lett. 41, 5361-5364 (2016).

8. J. Frauchiger, P. Albers, and H. P. Weber, "Modeling of thermal lensing and higher order ring mode oscillation in end-pumped C-W Nd:YAG lasers," IEEE J. Quantum Electron. 28, 1046-1056 (1992).

9. W. Koechner, Solid-State Laser Engineering, Springer Series in Optical Sciences (Springer New York, 2006), Vol. 1.

10. S. Chénais, F. Balembois, F. Druon, G. Lucas-Leclin, and P. Georges, "Thermal lensing in diode-pumped ytterbium lasers-Part I: theoretical analysis and wavefront measurements," IEEE J. Quantum Electron. 40, 1217-1234 (2004).

11. M. Schmid, T. Graf, and H. P. Weber, "Analytical model of the temperature distribution and the thermally induced birefringence in laser rods with cylindrically symmetric heating," J. Opt. Soc. Am. B 17, 1398-1404 (2000).

12. C. Pfistner, R. Weber, H. P. Weber, S. Merazzi, and R. Gruber, "Thermal beam distortions in end-pumped Nd:YAG, Nd:GSGG, and Nd:YLF rods," IEEE J. Quantum Electron. 30, 1605-1615 (1994).

13. A. E. Siegman, "Analysis of laser beam quality degradation caused by quartic phase aberrations," Appl. Opt. 32, 5893 (1993).

14. W. A. Clarkson, "Thermal effects and their mitigation in end-pumped solid-state lasers," Journal of Physics D: Applied Physics 34, 2381-2395 (2001).

15. F. Salin and J. Squier, "Gain guiding in solid-state lasers," Opt. Lett. 17, 1352 (1992).

16. A. Ritsataki, G. H. C. New, R. Melish, S. C. W. Hyde, P. M. W. French, and J. R. Taylor, "Theoretical modeling of gain-guiding effects in experimental all-solid-state KLM lasers," IEEE J. Sel. Top. Quantum Electron. 4, 185-192 (1998).

17. Z. Xiang, D. Wang, S. Pan, Y. Dong, Z. Zhao, T. Li, J. Ge, C. Liu, and J. Chen, "Beam quality improvement by gain guiding effect in endpumped Nd:YVO_4 laser amplifiers," Opt. Express 19, 21060 (2011).

18. X. Yan, Q. Liu, X. Fu, D. Wang, and M. Gong, "Gain guiding effect in endpumped Nd:YVO_4 MOPA lasers," J. Opt. Soc. Am. B 27, 1286 (2010). 
19. F. Sanchez, M. Brunel, and K. Aït-Ameur, "Pump-saturation effects in end-pumped solid-state lasers," J. Opt. Soc. Am. B 15, 2390-2394 (1998).

20. Y. Sato and T. Taira, "Saturation factors of pump absorption in solidstate lasers," IEEE J. Quantum Electron. 40, 270-280 (2004)

21. J. Didierjean, M. Castaing, F. Balembois, P. Georges, D. Perrodin, J. M. Fourmigué, K. Lebbou, A. Brenier, and O. Tillement, "High-power laser with $\mathrm{Nd}$ :YAG single-crystal fiber grown by the micro-pulling-down technique," Opt. Lett. 31, 3468 (2006).

22. I. Martial, F. Balembois, J. Didierjean, and P. Georges, "Nd:YAG singlecrystal fiber as high peak power amplifier of pulses below one nanosecond," Opt. Express 19, 11667 (2011).

23. X. Délen, S. Piehler, J. Didierjean, N. Aubry, A. Voss, M. A. Ahmed, T. Graf, F. Balembois, and P. Georges, "250 W single-crystal fiber Yb: YAG laser," Opt. Lett. 37, 2898-2900 (2012).

24. X. Délen, Y. Zaouter, I. Martial, N. Aubry, J. Didierjean, C. Hönninger, E. Mottay, F. Balembois, and P. Georges, "Yb:YAG single crystal fiber power amplifier for femtosecond sources," Opt. Lett. 38, 109 (2013).

25. F. Lesparre, J. T. Gomes, X. Délen, I. Martial, J. Didierjean, W. Pallmann, B. Resan, M. Eckerle, T. Graf, M. A. Ahmed, F. Druon, F. Balembois, and P. Georges, "High-power Yb:YAG single-crystal fiber amplifiers for femtosecond lasers in cylindrical polarization," Opt. Lett. 40, 2517 (2015).

26. F. Lesparre, J. T. Gomes, X. Délen, I. Martial, J. Didierjean, W. Pallmann, B. Resan, F. Druon, F. Balembois, and P. Georges, "Yb:YAG single-crystal fiber amplifiers for picosecond lasers using the divided pulse amplification technique," Opt. Lett. 41, 1628 (2016).

27. T. Y. Fan, "Heat generation in Nd:YAG and Yb:YAG," IEEE J. Quantum Electron. 29, 1457-1459 (1993).

28. D. S. Sumida and T. Y. Fan, "Effect of radiation trapping on fluorescence lifetime and emission cross section measurements in solid state laser media." Opt. Lett. 19, 1343 (1994).

29. Q. Liu, X. Fu, M. Gong, and L. Huang, "Effects of the temperature dependence of absorption coefficients in edge-pumped Yb:YAG slab lasers," J. Opt. Soc. Am. B 24, 2081-2089 (2007).

30. B. Chen, J. Dong, M. Patel, Y. Chen, A. Kar and M. A. Bass, "Modeling of high-power solid state slab lasers", Proc. SPIE 4968, p. 1-10 (2003)

31. S. Chénais, F. Druon, S. Forget, F. Balembois, and P. Georges, "On thermal effects in solid-state lasers: The case of ytterbium-doped materials," Prog. Quantum Electron. 30, 89-153 (2006).

32. C. Geuzaine and J.-F. Remacle, "Gmsh: A 3-D finite element mesh generator with built-in pre-and post-processing facilities," Int. J. Numer. Methods Eng. 79, 1309-1331 (2009).

33. O. C. Zienkiewicz, K. Morgan, Finite Elements and Approximation (John Wiley \& Sons, 1983)

34. R. Wynne, J. L. Daneu, and T. Y. Fan, "Thermal coefficients of the expansion and refractive index in YAG," Appl. Opt. 38, 3282 (1999).

35. S. Chenais, F. Druon, F. Balembois, P. Georges, A. Brenier, and G. Boulon, "Diode-pumped Yb: GGG laser: comparison with Yb: YAG," Opt. Mater. 22, 99-106 (2003).

36. A. Cousins, "Temperature and thermal stress scaling in finite-length end-pumped laser rods", IEEE Journal of Quantum Electronics 28, 1057 1069 (1992).

37. R. J. Noll, "Zernike polynomials and atmospheric turbulence*," J. Opt. Soc. Am. 66, 207 (1976).

38. J. D. Schmidt, Numerical Simulation of Optical Wave Propagation with Examples in MATLAB (SPIE, 2010).

39. D. G. Voelz, Computational Fourier Optics: $A$ MATLAB ${ }^{\circledR}$ Tutorial (SPIE, 2011).

40. J. W. Goodman, Introduction to Fourier Optics (Roberts and Company Publishers, 2005).

41. C. Hönninger, R. Paschotta, M. Graf, F. Morier-Genoud, G. Zhang, M. Moser, S. Biswal, J. Nees, A. Braun, G. A. Mourou, I. Johannsen, A. Giesen, W. Seeber, and U. Keller, "Ultrafast ytterbium-doped bulk lasers and laser amplifiers," Appl. Phys. B 69, 3-17 (1999).
42. J.-C. Chanteloup, "Multiple-wave lateral shearing interferometry for wave-front sensing," Appl. Opt. 44, 1559 (2005). 